\title{
Development of hepatic metastases in colorectal cancer - new insights
}

\author{
Pulathis N. Siriwardana, MBBS, MRCS, MS', Kemal I. Deen, MBBS, MS, MD, FRCS \\ 1 Assistant Surgeon, Department of HBP and Liver Transplant Surgery, Royal Free Hospital and Honorary \\ Clinical Lecturer, University College London Medical School, UK. \\ 2 Professor of Surgery, University of Kelaniya Medical School, Ragama, Sri Lanka.
}

\begin{abstract}
Introduction: Colorectal cancer (CRC) is the third most common malignancy in world. One third of all treated patients continue to develop hepatic metastases. Understanding the mechanism of spread and the establishment of metastases in the liver is vital in planning potentially curative treatment strategies for these patients.

Methods: A Pubmed and Medline literature search using keywords and their variations of colorectal, rectal, colon, cancer, hepatic metastasis and mechanisms was performed. Since all the retrieved mechanisms could not be included in this review, the search was further refined by using terms in the multistep process of hepatic metastasis of CRC.

Results: The initial search retrieved 2474 articles. Seven mechanisms were identified for the review by performing a highly selective search. They were based on the multistep process of hepatic metastasis, recently discovered pathophysiological and related molecular factors. These were, proteolytic enzymes, venous invasion, circulating tumour cells, evasion of host immune system, adhesion molecules, angiogenesis and tumour hypoxia

Conclusion: The search identified a wide range of molecular mechanisms contributing to the metastatic process of CRC to the liver. Their inclusion in this review was based on the authors' selection criteria with a high probability of selection bias. Nevertheless, an understanding of the complex molecular mechanisms of developing hepatic metastases would enhance the ability to treat patients in view of prevention of disease progression to liver and improving overall outcome.
\end{abstract}

Key words: Colorectal Cancer; Hepatic Metastases; Molecular Mechanisms.

\section{Background}

Colorectal cancer (CRC) is the second cause of cancer deaths in North America and Western Europe [1]. With increased awareness and effective screening tools more patients present with early disease, resulting in high resectability and a potential cure. Despite having a curative resection (R0), 30 to $50 \%$ of patients will die within 5 years due to metastatic disease and local recurrence [2]. Hepatic metastases account for up to $44 \%$ of these patients [3]. Hence, prognosis and overall survival are determined mainly by the

Correspondence: Pulathis Siriwardana, Dept of HBP and Liver

Transplant Surgery, Royal Free Hospital, 9th Floor, Royal Free Hospital, Pond Street, London, NW3 2QG, UK

Email: pulathis_ns@yahoo.co.uk

The Sri Lanka Journal of Surgery 2012; 30(1):13-17. progression of colorectal liver metastases (CRLM) and if remain untreated, life expectancy is less than a year [4].

The contemporary paradigm of development of hepatic metastases in CRC is based on a multistep model. This is defined by a series of progressive somatic genetic alterations which give malignant cells the ability to progress through many phases of metastasis. The common processes involved are, proteolysis of local extracellular matrix (ECM) attachments, adhesive alterations and invasion, neovascularisation, viable vascular dissemination, host immune evasion, tumour embolisation, adhesion and survival in a host environment. 
A comprehensive analysis of all mechanisms is not possible due to the vast amount of published research. The focus of this review is to outline the important, most recent and most frequently studied mechanisms involved in the development of CRLM.

\section{Proteolytic enzymes}

It is prudent to hypothesise that detachment of cells from the primary tumour is the initial step of the multistep process of distant metastasis. Proteolytic enzymes play a pivotal role in the degradation of extracellular matrix components and regulation of cellular functions by cleaving surface proteins, enabling many tumour cells to detach from their primary site and invade surrounding tissue and vessels. There are many classes of proteinases associated with the development of metastases including serine, cystine, aspartic and matrix metalloproteinases (MMP), whilst some studies suggest the latter exerts a more dominant role [5]. The most studied proteinases in relation to formation of CRLM are MMP7 and UPA. MMP7 is the smallest in this group. It is implicated as the most important proteinase since it is over expressed in a majority of CRCs and its expression is associated with hepatic metastases [6]. Urokinase Plasminogen Activator (uPA) is a serine protease activated by the uPA receptor (UPAR). Activated uPA converts plasminogen into plasmin. This mediates extracellular matrix degradation and activation of pro MMPs located in the extracellular space. Whilst, high uPAR expression in CRC expression has been correlated with decreased 5 year survival [7], experimental data provides evidence for its role in the development of hepatic metastases [8].

\section{Venous Invasion}

This is an important prognostic indicator in colorectal cancer and is associated with an increased risk of visceral metastasis and reduced overall survival [9]. Approximately $55 \%$ of all CRCs show histological evidence of venous invasion. However, in the cohort of CRCs with synchronous liver metastases, the rate of venous invasion is increased to $90 \%$ [10]. This data is in keeping with the results of Mori et al. who reported venous invasion in $91 \%$ and $95 \%$ of CRCs with synchronous and metachronous liver metastases respectively, compared to $44.2 \%$ in those with no metastases [11].

\section{Circulating tumour cells}

Tumour dissemination through the circulation is an essential step in the development of CRLM. In a recent meta-analysis, metachronous hepatic metastases were significantly higher $(21 \%)$ in patients with CRC who had circulating cancer cells at the time of resection, compared to those who did not [12]. Hence, circulating tumour cells could be used as a prognostic marker and probably an aid to plan adjuvant therapy for colorectal cancer patients. It may be more important in stage I and II colorectal cancer where the role adjuvant chemotherapy is less defined. The contribution of surgical handling in tumour metastasis via the portal circulation has been a much debated topic. This is important in planning surgical resection of the primary cancer. The 'no touch isolation' technique, in which lymphovascular ligation is performed before manipulation of the primary CRC was proposed by Barnes in 1952 [13], and an early proponent of this was Turnbull et al. [14]. However, their results have been refuted by prospective trials in the past. With the advancement of newer molecular biology techniques, researchers have revisited this concept. Atkin et al. reported a trend towards reduced tumour cell dissemination for the no touch isolation technique but failed to determine its clinical significance [15]. Nevertheless, the 'no touch' technique could be recommended during the resection of the primary tumour whenever possible.

\section{Evasion of host immune system}

Normal cells as opposed to tumour cells are typically susceptible to the host immune system or undergo apoptosis in an anchorage independent environment and away from the primary site. There are many molecular factors that provide colorectal cancer cells the ability to survive in circulation and in the hepatic environment. The ability of colon cancer cells to survive in an anchorage free environment and form hepatic metastases is facilitated by MMP7 (Matrilysin), which induces an E-cadherin mediated aggregation process [6]. Metastasised CRC also has the ability to evade hepatic Natural Killer (NK) cell destruction by differential expression or non expression of receptors and decoys. Deficiencies of the host immune defence mechanism are also known to increase the incidence of hepatic metastasis of CRC. Interferon $\beta$ (IFN $\beta$ ) is a cytokine with an anti metastatic effect on many human cancers and has been shown to play an important role in gene expression necessary to prevent hepatic metastasis in 
CRC cells [16]. Development of CRLM is linked to the lack of IFN $\beta$ in the patient and also resistance of the primary CRC to IFN $\beta$.

\section{Adhesion molecules}

Viability of many tissues including cancers is dependent on cell adhesion. Disruption of cell adhesions is a preliminary step in tumour dissemination. Promotion and maintenance of these adhesions are important processes in the development of metastatic lesions. Multiple cell adhesion molecules (CAM) play an important role in detachment of metastatic cells from the primary carcinoma and attachment to distant site. Whilst CAMs are down regulated in the former case, in the latter, cell surface molecules mediate tumour-host attachment [17]. The hepatic colonisation of CRC is initiated in the sinusoids where sinusoidal endothelial cells (SECs), Kupffer cells and stellate cells interact with the metastasised cells which express a variety of CAMs and their receptors. The classes of adhesion molecules implicated in the development of CRLM include integrins, cadherins, selectins, immunoglobulins and hyaluronate binding proteins. The mostly studied adhesion molecules are integrins and cadherins. Integrins of CRC can bind to many extracellular matrix (ECM) molecules including laminin and collagen. Staining experiments have shown strong expression of laminin molecules in the cytosols of luminal epithelial cells of portal and hepatic veins and in the normal liver. Hence, expression of integrins in CRC specific to laminins of the portal and hepatic vessels mediates preferential development of hepatic metastases. Extensive experimental and clinical data show the association of the cadherin-catenin system with CRC and hepatic metastasis. The clinical stage, liver metastasis, tumour recurrence and five year overall survival is significantly correlated with the expression of high levels of E-cadherin in CRC [18].

\section{Angiogenesis}

Angiogenesis is crucial for the outgrowth of the primary cancer and this becomes a source of haematogenous spread. It is also important in the establishment and progression of CRLM. Diffusion of oxygen alone becomes inadequate in maintaining the viability of tumours that are more than $1-2 \mathrm{~mm}$ in diameter. The main factor stimulating angiogenesis in tumours is Vascular Endothelial Growth Factor (VEGF). It acts directly on the endothelial cells as a mitogen to induce cell migration, proliferation, invasion and increased cell permeability [19]. Increased VEGF in the primary is associated with poor prognosis and it has been found to be important in the formation of hepatic metastasis [20]. Interestingly a few studies have shown VEGF to be up-regulated in the primary whilst down-regulated in hepatic metastasis [21]. Although not precisely known, this may be due to intra-tumoral hypoxia of the primary, which is believed to be abolished in metastases within the highly vascularised liver parenchyma. However, hypoxia in CRLM has not been studied adequately.

\section{Tumour hypoxia}

Hypoxia is a common phenomenon in solid tumours and is known to cause more malignant phenotypes [22]. It occurs when the oxygen demand of the tumour exceeds the supply from its vasculature. Aberrant blood vessel formation, fluctuations in blood flow and increasing demand from rapid tumour expansion have been implicated in the occurrence of areas of hypoxia within tumours. Tumour cells subjected to hypoxia undergo a series of modifications enabling their survival and proliferation through activation of several genes. This is primarily achieved through activation of hypoxia-inducible factor - $1 \alpha$ (HIF-1 $\alpha$ ) which plays a key role in cellular adaptation to hypoxia in solid tumours by stimulating angiogenesis and glycolysis [23]. There is a statistically significant correlation between HIF- $1 \alpha$ expression and tumour invasion, lymphatic invasion, venous invasion and liver metastasis in colorectal cancer [24]. Rajaganeshan et al reported an increased incidence of metachronous liver metastases when the primary CRC had up-regulation of HIF-1 $\alpha$ [25]. Blocking HIF- $1 \alpha$ pathways may be a future therapeutic option not only to reduce the metastatic potential but also as a modality to reduce the aggressiveness of primary colorectal cancer.

\section{Conclusion}

Development of hepatic metastases is a significant hallmark of disease progression and an ominous event in the natural history of colorectal cancer. Despite having a curative resection, a significant proportion of patients continue to develop hepatic metastasis. The unique anatomy of the splanchnic circulation has enabled clinicians to focus surveillance and predict sites of metastasis. However, with advances in molecular biology techniques, new mechanisms of metastasis have been discovered clarifying the series of events occurring in the progression of CRC. These 
novel tools would in future help clinicians detect primary tumours with a high metastatic index and may expand the criteria for existing adjuvant treatment regimens. Targeting specific molecular pathways is the basis of most chemotherapeutic agents. With the advancement of molecular technologies elucidating the mechanism of hepatic metastasis more treatment options would be available. Hence understanding this intricate process would lead to better prognostication, detection and treatment strategies leading to increased survival of patients with CRC.

This review looks at some of the most frequently studied molecular mechanisms of hepatic metastasis of $\mathrm{CRC}$ and attempts to give some insight into a complex process.

\section{References}

1. McMillan DC, McArdle CS. Epidemiology of colorectal liver metastases. Surg. Oncol. 2007; $16: 3-5$.

2. Safi F, Beyer HG. The value of follow-up after curative surgery of colorectal carcinoma. Cancer Detect Prev. 1993;17:417-24.

3. Boring CC, Squires TS, Tong T. Cancer statistics, 1993. CA Cancer J Clin. 1993;43:7-26.

4. McLoughlin JM, Jensen EH, Malafa M. Resection of colorectal liver metastases: current perspectives. Cancer Control. 2006;13:32-41.

5. Zucker S, Vacirca J. Role of matrix metalloproteinases (MMPs) in colorectal cancer. Cancer Metastasis Rev. 2004;23:101-17.

6. Kioi M, Yamamoto K, Higashi S, Koshikawa N, Fujita K, Miyazaki K. Matrilysin (MMP-7) induces homotypic adhesion of human colon cancer cells and enhances their metastatic potential in nude mouse model. Oncogene. 2003;22:8662-70.

7. Ganesh S, Sier CF, Heerding MM, Griffioen G, Lamers CB, Verspaget HW. Urokinase receptor and colorectal cancer survival. Lancet. 1994;344:401-2.

8. Ahmed N, Oliva K, Wang Y, Quinn M, Rice G. Downregulation of urokinase plasminogen activator receptor expression inhibits Erk signalling with concomitant suppression of invasiveness due to loss of uPAR-beta1 integrin complex in colon cancer cells. Br J Cancer. 2003;89(2):374-84.

9. Meguerditchian AN, Bairati I, Lagacé R, Harel F, Kibrité A. Prognostic significance of lymphovascular invasion in surgically cured rectal carcinoma. Am J Surg. 2005; 189:707-13.

10. Ouchi K, Sugawara T, Ono H, Fujiya T, Kamiyama Y, Kakugawa Y, Mikuni J, Tateno H. Histologic features and clinical significance of venous invasion in colorectal carcinoma with hepatic metastasis. Cancer. 1996;78(11):2313-7.

11. Mori D, Shibaki M, Masuda M, Yamasaki F. Quantitative measurement of venous invasion of colorectal cancer with metachronous liver metastasis. Histopathology. 2009;55:654-9.

12. Katsuno H, Zacharakis E, Aziz O, Rao C, Deeba S, Paraskeva P, Ziprin P, Athanasiou T, Darzi A. Does the presence of circulating tumor cells in the venous drainage of curative colorectal cancer resections determine prognosis? A metaanalysis. Ann Surg Oncol. 2008;15:3083-91.

13. Barnes JP. Physiologic resection of the right colon. Surg Gynecol Obstet. 1952;94:722-6.

14. Turnbull RB Jr, Kyle K, Watson FR, Spratt J. Cancer of the colon: the influence of the notouch isolation technique on survival rates. Ann Surg. 1967;166(3):420-7.

15. Atkin G, Chopada A, Mitchell I. Colorectal cancer metastasis: in the surgeon's hands? Int Semin Surg Oncol. 2005;2:5.

16. Zimmer R, Thomas P. Expression profiling and interferon-beta regulation of liver metastases in colorectal cancer cells. Clin Exp Metastasis. 2002;19:541-50.

17. Haier J, Nasralla M, Nicolson GL. Cell surface molecules and their prognostic values in assessing colorectal carcinomas. Ann Surg. 2000;231(1):11-24.

18. Dorudi S, Hanby AM, Poulsom R, Northover J, Hart IR. Level of expression of E-cadherin mRNA in colorectal cancer correlates with clinical outcome. Br J Cancer. 1997;7:614-6.

19. Nicosia RF. What is the role of vascular endothelial growth factor-related molecules in tumor angiogenesis? Am J Pathol. 1998;153(1):11-6.

20. Takahashi Y, Kitadai Y, Bucana CD, Cleary KR, Ellis LM. Expression of vascular endothelial growth factor and its receptor, KDR, correlates with vascularity, metastasis, and proliferation of human colon cancer. Cancer Res. 1995;55(18):3964-8.

21. Jung YD, Ahmad SA, Akagi Y, Takahashi Y, Liu W, Reinmuth N, Shaheen RM, Fan F, Ellis LM. Role of the tumor microenvironment in mediating response to anti-angiogenic therapy. Cancer Metastasis Rev. 2000;19:147-57.

22. Semenza, G. L. Involvement of hypoxia- 
inducible factor 1 in human cancer. Internal Medicine, 2002;41:79-83.

23. Semenza GL. Targeting HIF-1 for cancer therapy. Nat Rev Cancer. 2003;3:721-32.

24. Kuwai T, Kitadai Y, Tanaka S, Onogawa S, Matsutani N, Kaio E, Ito M, Chayama K. Expression of hypoxia-inducible factor-1alpha is associated with tumor vascularization in human colorectal carcinoma. Int $\mathrm{J}$ Cancer. 2003;105:176-81.

25. Rajaganeshan R, Prasad R, Guillou PJ, Poston $\mathrm{G}$, Scott N, Jayne DG. The role of hypoxia in recurrence following resection of Dukes' B colorectal cancer. Int $\mathrm{J}$ Colorectal Dis. 2008;23:1049-55. 